\title{
Eficacia de un programa grupal, online sincrónico de salud y bienestar para personas que trabajan con jóvenes y adultos con discapacidad intelectual
}

\section{Effectiveness of a synchronous online, group health and wellness program for people who work with youths and adults with intellectual disabilities}

\author{
Flavia Arrigoni \\ Universidad del Aconcagua, Mendoza, Argentina \\ Mara Abraham \\ Centro de Día "Mundo Alas", Mendoza, Argentina \\ Ivana Polo \\ Universidad del Aconcagua, Mendoza, Argentina
}

\section{Resumen}

Fundamentos. Se diseñó e implementó un programa de promoción de la salud destinado a personas que trabajan con jóvenes y adultos con discapacidad intelectual. Los objetivos perseguidos fueron evaluar la eficacia de un programa de promoción de la salud grupal, online sincrónico y promover el bienestar de los trabajadores. Método. Se trata de una investigación con un enfoque cuantitativo, exploratorio descriptivo e interactivo, con un diseño cuasiexperimental, pre pos test para muestras pareadas. Instrumentos: Cuestionario de Salud General (GHQ 12), Índice de Felicidad de Pemberton, Maslach Burnout Inventory (MBI), Inventario de ansiedad de Beck (BAI) y una ficha sociodemográfica ad hoc. El programa de intervención desarrollado, "Bienestar y salud”, constó de 8 sesiones grupales, online, sincrónicas (por Google meet), de frecuencia semanal, con una duración de 120 minutos. La participación fue voluntaria y firmaron un consentimiento informado. Resultados. Participaron un total de 35 trabajadores con una edad promedio de 31.11 años. Tras la intervención, se observó un incremento en el bienestar personal y la realización personal en el trabajo y una reducción en el nivel de malestar psicológico, ansiedad, agotamiento emocional y despersonalización. Se observaron diferencias estadísticamente significativas en las puntuaciones pre pos test del malestar psicológico y de la dimensión agotamiento emocional del MBI. Conclusión. Los hallazgos permiten concluir que participar en un programa de promoción de la salud en formato grupal en línea sincrónico resultó eficaz para incrementar el bienestar y la salud de los trabajadores.

Palabras clave: promoción de la salud, telepsicología, confinamiento, COVID-19, personal que trabaja con personas con discapacidad intelectual

Flavia Arrigoni; Universidad del Aconcagua, Argentina; Mara Abraham, Centro de Día "Mundo Alas", Argentina; Ivana Polo, Universidad del Aconcagua, Argentina.

La correspondencia en relación con este artículo se dirige a Flavia Arrigoni, Universidad del Aconcagua, Argentina. Correo electrónico: farrigoni@uda.edu.ar 


\begin{abstract}
Background. A health promotion program was designed and implemented for people who work with youths and adults with intellectual disabilities. The objectives were to evaluate the effectiveness of a synchronous online group health promotion program and to promote worker well-being. Method. It is an investigation with a quantitative, descriptive and interactive approach, with a quasi-experimental design, pre- and posttest for paired samples. Instruments: General Health Questionnaire (GHQ 12), Pemberton Happiness Index, Maslach Burnout Inventory (MBI), Beck Anxiety Inventory (BAI) and an ad hoc sociodemographic form. The intervention program carried out, "Well-being and health", consists of 8 group synchronous online sessions (on Google meet), with a weekly frequency, lasting 120 minutes. Results. Participation was voluntary and all signed an informed consent. A total of 35 workers with an average age of 31.11 years participated. After the intervention, there was an increase in personal well-being and personal fulfillment at work, and a reduction in the level of psychological discomfort, anxiety, emotional exhaustion and depersonalization. Statistically significant differences were observed in the scores preand posttest of psychological distress and emotional exhaustion. Conclusion. The results allow us to conclude that participating in a synchronous online group health promotion program was effective to increase the well-being and health of workers.
\end{abstract}

Keywords: Health promotion, Telepsychology, Lockdown, COVID-19, Workers with people with intellectual disabilities

El 11 de marzo de 2020, la Organización Mundial de la Salud (2020) declaró como pandemia al brote del SARS-CoV-2/COVID-19. En Argentina, el 20 de marzo de ese año, el Poder Ejecutivo Nacional dispuso el aislamiento social, preventivo y obligatorio como una medida para prevenir los contagios y mitigar la circulación del virus en el país.

A partir de ese momento, todas las instituciones educativas, y considerando como tales a los centros de días que acompañan a jóvenes y adultos con discapacidad intelectual, se vieron obligadas a reorganizar su quehacer para garantizar la continuidad del servicio prestado de manera no presencial.

La situación de alerta sanitaria implicó planificar propuestas de intervención para promover la salud de los trabajadores sin que esto implicara la presencialidad. Las propuestas online se presentaron como adecuadas para favorecer el cuidado de la salud y el establecimiento de relaciones interpersonales con colegas y compañeros de trabajo (Arón, 2016), tan necesarias en esa situación tan particular.

El clima de incertidumbre, la creciente cifra de muertes en el país y el mundo, la diversidad de información desde múltiples y contradictorias fuentes, la pertenencia a algún grupo de riesgo, sumado a ciertos rasgos de personalidad, son solo algunas de las circunstancias que permiten explicar por qué la pandemia y las medidas de confinamiento pueden haber impactado en el estatus de salud y bienestar de las personas.

Sanclemente et al. (2020) han señalado el vínculo existente entre períodos de crisis económica con la satisfacción, la inseguridad y la salud laboral. Si a esta crisis sanitaria se le añade la crisis económica de Argentina y el consecuente incremento de la inseguridad laboral, es posible inferir cierto malestar, inseguridad, vulnerabilidad e indefensión (Blanch, 2011), así como también una reducción de la salud física y psicológica de los trabajadores (De Witte, 2016). 
El aislamiento implicó cambios radicales en la rutina de las personas al generar estrés, miedo, ansiedad, entre otras experiencias emocionales y resultó en un incremento del malestar psicológico y una disminución del afecto positivo y bienestar general (Consejo General de la Psicología de España, 2020). Urgió la necesidad de diseñar e implementar programas de promoción de la salud útiles para crear condiciones personales y sociales que favorezcan el desarrollo psicológico y psicofisiológico de la persona (Hosman et al., 2005).

Debido al creciente auge de internet y las nuevas tecnologías de la información y comunicación (TICs), el Colegio Oficial de Psicología de Madrid, en colaboración con el Centro de Psicología Aplicada de la Universidad Autónoma de Madrid, editaron la Guía para la Intervención en Tele-psicología (De la Torre-Martí \& Pardo-Cebrián, 2018), que regula la oferta de servicios psicológicos.

Durante la pandemia, la Federación de Psicólogos de la República Argentina recomendó el uso de formas alternativas para ofrecer terapia psicológica. Su Guía de Intervención en Telepsicología (FEPRA, 2021) regula la oferta de servicios psicológicos a través de las TICs; por ejemplo, las videoconferencias interactivas se consideraron una alternativa viable para ofrecer terapia psicológica, porque se basan en la interacción de paciente y terapeuta en una plataforma virtual.

La American Psychological Association (2013) considera como telepsicología a todo tipo de servicio psicológico que implique o utilice las TICs. Se incluyen teléfonos celulares, computadoras, videoconferencias interactivas, el correo electrónico, entre otros (Consejo General de Psicología de España, 2017). Al respecto, Cabas-Hoyos (2020) señala que los servicios de telepsicología:

son también denominados e-terapia, consulta online, terapia virtual o ciberterapia, y abarcan desde intercambios de correo electrónico hasta conversaciones por chat, videollamadas o notas de voz. Estas herramientas han sido utilizadas por más de 16 años, tanto de forma individual como grupal (p. 43).

Jané-Llopis (2004) señala que "Las intervenciones de prevención y promoción en salud mental son eficaces y se han traducido en beneficios para la salud y la sociedad" (p.67) al mejorar la calidad de vida de la persona. Barlow et al (2019) entienden que las personas utilizan estrategias de regulación emocional que, paradójicamente, contribuyen al mantenimiento o empeoramiento de los síntomas que pretenden erradicar: "todo el mundo puede beneficiarse de aprender maneras saludables de responder a sus emociones" (p.25). Consideran la conveniencia de incorporar nuevas formas de gestión de las propias emociones cuando estas interfieren en el propio bienestar y enfatizan el rol activo de la persona en el restablecimiento de su salud y bienestar.

La propuesta de intervención aplicada y evaluada se enmarca en una perspectiva positiva de salud, desde la cual se contempla diseñar e implementar intervenciones psicológicas que promuevan el autocuidado y factores protectores que aporten al bienestar general de las personas (Rosen et al., 2020). La Psicología Positiva se interesa por el estudio sobre el rol y promoción de las características positivas que pueden impactar en el bienestar de las personas (Wood \& Joseph, 2010). Se trató de un programa telemático en el ámbito de la salud mental, y es importante considerar que los "I Care program" (Beecham et al., 2019), se presentaron como una eficaz estrategia de intervención en un contexto de restricciones debido a la situación de alerta sanitaria. 
Los objetivos generales fueron los siguientes: evaluar la eficacia de un programa de promoción de la salud grupal, online sincrónico y promover el bienestar de personas que trabajan con jóvenes y adultos con discapacidad intelectual; y los objetivos específicos: (a) Incrementar el bienestar y disminuir el malestar psicológico, (b) Disminuir el nivel de ansiedad y (c) Disminuir el nivel de agotamiento emocional y la despersonalización en el trabajo e incrementar la realización personal en el trabajo.

\section{Método}

Se trata de una investigación con un enfoque cuantitativo, exploratorio-descriptivo e interactivo, con un diseño cuasiexperimental, pre pos test para muestras pareadas (Hernández-Sampieri \& Mendoza, 2018) desarrollada durante los meses de agosto-diciembre del año 2020.

En la perspectiva cuasi-experimental, "el criterio de asignación de los sujetos o unidades a las condiciones de tratamiento o condiciones de estudio no se rige por las leyes del azar" (Arnau \& Gómez, 1995, p. 15). Se realizaron 2 mediciones repetidas (pre test y pos test) de muestras pareadas o correlacionadas, de manera tal que la observación pos estuvo relacionada con la observación pre. Se optó por un diseño pareado tipo 2 , porque se cuenta con medidas tomadas a una misma unidad de análisis en dos tiempos distintos.

\section{Participantes}

Los participantes fueron 35 trabajadores de dos "Centros de día” del Gran Mendoza, Argentina, con una edad promedio de 31.11 años.

Del total de 35 trabajadores, el 86\% eran mujeres y el 14\% restante, varones. En términos generales, los participantes fueron en su mayoría mujeres, solteras, sin hijos, con una formación universitaria. Los datos sociodemográficos que se tomaron de los participantes se resumen en la tabla 1. 
EFICACIA DE UN PROGRAMA GRUPAL, ONLINE SINCRÓNICO DE SALUD Y BIENESTAR

\section{Tabla 1}

Frecuencias y porcentajes de los datos sociodemográficos generales tomados de los participantes

\begin{tabular}{|c|c|c|c|}
\hline \multicolumn{2}{|l|}{ Dato } & \multirow{2}{*}{$\frac{\text { Frecuencia }}{30}$} & \multirow{2}{*}{$\begin{array}{c}\% \\
86 \%\end{array}$} \\
\hline Género & Mujer & & \\
\hline & Varón & 5 & $14 \%$ \\
\hline \multirow[t]{5}{*}{ Estado civil } & Soltero/a & 17 & $48.5 \%$ \\
\hline & Casado/a & 6 & $17 \%$ \\
\hline & Concubino/a & 10 & $28.5 \%$ \\
\hline & Viudo/a & 1 & $3 \%$ \\
\hline & Divorciado/a & 1 & $3 \%$ \\
\hline \multirow[t]{4}{*}{ N..$^{o}$ de hijos } & 0 & 23 & $66 \%$ \\
\hline & 1 & 4 & $11 \%$ \\
\hline & 2 & 6 & $17 \%$ \\
\hline & $>2$ & 2 & $6 \%$ \\
\hline \multirow[t]{4}{*}{ Convivencia } & Solo & 3 & $8.5 \%$ \\
\hline & Con pareja e hijos & 17 & $48.5 \%$ \\
\hline & Con otros familiares & 12 & $34.5 \%$ \\
\hline & Otro & 3 & $8.5 \%$ \\
\hline \multirow[t]{5}{*}{ Nivel de instrucción } & Secundarios & 3 & $8.5 \%$ \\
\hline & Terc. Incompleto & 3 & $8.5 \%$ \\
\hline & Terc. Completo & 6 & $17 \%$ \\
\hline & Univ. Incompleto & 11 & $31.5 \%$ \\
\hline & Univ. Completo & 12 & $34.5 \%$ \\
\hline \multirow[t]{5}{*}{ Profesión / ocupación } & Estudiante & 17 & $48.5 \%$ \\
\hline & Psicóloga/o & 4 & $11 \%$ \\
\hline & Trabajador social & 3 & $8.5 \%$ \\
\hline & Educador & 6 & $17 \%$ \\
\hline & Otro & 5 & $14.5 \%$ \\
\hline \multirow[t]{6}{*}{ Rol en la institución } & Tallerista & 11 & $31.5 \%$ \\
\hline & Orientador & 13 & $37.5 \%$ \\
\hline & Auxiliar & 4 & $11 \%$ \\
\hline & Administrativo & 1 & $3 \%$ \\
\hline & Gestión & 2 & $6 \%$ \\
\hline & Técnico & 4 & $11 \%$ \\
\hline
\end{tabular}




\section{Variables}

Variable independiente: se aplicó el programa "Bienestar y Salud" (Arrigoni, 2021), el cual constó de ocho sesiones grupales, online, sincrónicas (desarrolladas a través de la plataforma Google meet), de frecuencia semanal, con una duración de 120 minutos. Las temáticas seleccionadas para cada una de las ocho sesiones fueron: (a) Orientaciones psicológicas durante el aislamiento, (b) Estilo de vida saludable, (c) Psicología positiva, (d) Flexibilidad cognitiva, (e) Gestión de las emociones, (f) Higiene del sueño, (h) Estrés y estrategias de afrontamiento y (i) Lineamientos para el autocuidado y el cuidado del equipo de trabajo. Durante las sesiones, se asignaron tareas para la casa (autorregistro de emociones, práctica de relajación, entre otras). La principal estrategia fue la psicoeducación. Se trata de un programa de 24 horas de duración de las cuales 16 horas fueron en línea en tiempo real y 8 horas de tareas para la casa. Para su diseño, se tomó como base el "Programa Salud y bienestar" desarrollado durante el confinamiento desde la Universidad de Cádiz (Arrigoni et al., 2020) y el "Programa PARE" destinado a personas que trabajan con jóvenes y adultos con discapacidad intelectual (Arrigoni \& Fadin, 2019; Arrigoni et al., 2021). Buscó favorecer la adquisición de un estilo de vida saludable y el entrenamiento en técnicas de relajación con el fin de proteger a los trabajadores, no solo del impacto de la ansiedad y del estrés asociados al confinamiento, sino también como estrategias de autocuidado eficaces para un óptimo desempeño del trabajo institucional (Arrigoni, 2021).

Variables dependientes:

- Malestar psicológico: estado de distrés caracterizado por ausencia de bienestar subjetivo; presencia de síntomas ansiosos y depresivos; afectación del nivel de funcionamiento general (relaciones interpersonales y el nivel de funcionamiento cotidiano) y riesgo de cometer agresiones a terceros o autolesiones.

- Bienestar subjetivo: constructo que incluye un componente afectivo, presencia de emociones positivas y ausencia de emociones negativas, y otro cognitivo, relacionado con la satisfacción con la vida (Vera-Villarroel et al., 2012).

- Ansiedad: hiperactivación fisiológica "como una respuesta anticipatoria a una amenaza futura que puede existir o no” (Pineda-Sánchez, 2018, p. 30).

- Dimensiones del síndrome de burnout: agotamiento emocional, despersonalización y realización personal en el trabajo. El Agotamiento Emocional (AE) alude al sentimiento del trabajador de que se han agotado sus recursos emocionales. La Despersonalización (D) supone actitudes negativas, insensibles, distantes, e incluso cínicas hacia los destinatarios de una labor profesional. La Realización Personal (RP) se relaciona con la experiencia de satisfacción personal en el trabajo y la percepción de posibilidades de promoción personal. Altas puntuaciones en las subescalas AE y D; y bajas puntuaciones en la escala RP muestran un alto grado de burnout o malestar.

\section{Instrumentos}

Con el fin de evaluar la eficacia del programa implementado, se consideró propicio medir las siguientes variables: malestar psicológico, bienestar, ansiedad y las tres dimensiones del síndrome de quemarse por el trabajo: agotamiento emocional, despersonalización y realización personal en el trabajo. Los instrumentos seleccionados para su valoración fueron los siguientes: 
EFICACIA DE UN PROGRAMA GRUPAL, ONLINE SINCRÓNICO DE SALUD Y BIENESTAR

Cuestionario de Salud General (GHQ-12) de Goldberg y Williams (1988), validado en Argentina (Burrone et al., 2015). Es un instrumento autoadministrado de cribado que tiene por objetivo detectar morbilidad psicológica y posibles casos de trastornos psiquiátricos. Está compuesto por 12 ítems, 6 de ellos corresponden a sentencias positivas y 6 sentencias negativas. Los ítems son contestados mediante una escala tipo Likert de cuatro puntos (0-1-2-3), que puede ser transformada en una puntuación dicotómica (0-0-1-1), llamada puntuación GHQ. El GHQ-12 presenta una buena fiabilidad en un estudio desarrollado en Argentina, "ya que describe un alfa de Cronbach de 0,80" (Burrone et al., 2015, p.236). De la sumatoria de las puntuaciones dicotómicas obtenidas en todos los enunciados de la escala, se obtiene la puntuación de GHQ, de manera tal que, a medida que esta aumenta, disminuye el nivel de salud mental. A partir de la clasificación de Fullerton et al. (2003), "se consideran los valores de 0 a 4 del GHQ como indicadores de ausencia de psicopatología, de 5 a 6 como psicopatología subumbral y de 7 a 12 puntos como indicativos de presencia de psicopatología" (Burrone et al., 2015, p.238). Menores puntuaciones reflejan menor malestar psicológico.

Índice de Felicidad de Pemberton-PHI. El PHI es de dominio público, sin derechos de autor registrado. Fue traducido al español y validado por Hervás y Vázquez (2013). La versión validada en Argentina evalúa bienestar y afectividad positiva (Delfino et al., 2019). La persona debe realizar una evaluación retrospectiva respecto a diversas áreas de su vida. Incluye tanto el bienestar recordado como el experimentado (el día de ayer), aunque tales mediciones pueden hacerse por separado. El PHI recordado contiene un total de 11 ítems que valoran el bienestar en distintos ámbitos (general, eudaimónico, hedonista y social) y 10 ítems relacionados con el bienestar experimentado el día de ayer. Al considerar lo propuesto por Delfino et al. (2019, p. 118), se utilizó "únicamente la dimensión recordada del bienestar sin considerar el ítem relativo a emocionalidad negativa (ítem 10)". De esta forma, se obtiene un alfa de Cronbach $=$ .88 (10 ítems). Para su cálculo, se realiza el sumatorio de las puntuaciones obtenidas en cada uno de los 10 ítems. Las opciones de respuesta de cada ítem van de 0 a 10 , donde 0 corresponde a totalmente en desacuerdo y 10 a totalmente de acuerdo. Así, se obtiene un rango de puntuación de 0 a 100 donde mayor puntuación se corresponde con un mayor bienestar.

Inventario de Ansiedad de Beck - BAI. Se utilizó la versión traducida al español del Beck Anxiety Inventory (Beck \& Steer, 2011), cuya adaptación argentina ha mostrado ser un instrumento válido y confiable para la evaluación de la gravedad asociada a la ansiedad durante la última semana (Vizioli \& Pagano, 2020). Autoinforme de 21 ítems con una escala de respuesta de tipo Likert de cuatro puntos que va de " $0=$ Para nada" a " 3 = Severamente". La puntuación mínima es 0 y la máxima 63 . Vizioli y Pagano (2020) obtuvieron los siguientes índices de fiabilidad: $\alpha$ ordinal $=0.93, \omega$ ordinal $=0.95$, y confiabilidad compuesta $\rho=.92$.

Maslach Burnout Inventory (MBI) (Maslach \& Jackson, 1981, adaptación argentina de Hein, 2005). Cuestionario autoadministrable de 22 reactivos presentados como afirmaciones sobre posibles actitudes y sentimientos que mantiene el trabajador hacia su trabajo y los usuarios de su servicio. Evalúa tres subescalas: Agotamiento Emocional (AE), Despersonalización (D) y Realización Personal (RP). Los ítems son contestados a través de una escala tipo Likert de siete puntos que va de " $0=$ Nunca" a " $6=$ Todos los días". Para la corrección del cuestionario, se suma la puntuación directa obtenida en los ítems que corresponden a cada subescala. Altas puntuaciones en AE y D y una baja puntuación en la subescala 
RP confirman un alto grado de burnout (SBO). Las puntuaciones se clasifican mediante un sistema de percentil para cada escala. Los análisis de confiabilidad del instrumento arrojan valores aceptables: alfa de Cronbach $=.86$ para la escala general (Casari et al., 2017).

Ficha sociodemográfica ad hoc. Incluye ciertas variables sociodemográficas edad, género, estado civil, número de hijos, nivel de estudios, profesión/ocupación y situación laboral actual.

\section{Procedimiento}

La muestra del estudio se obtuvo partir de la convocatoria efectuada a dos Centros de día de la Provincia de Mendoza (Argentina). Los centros de días son instituciones educativas que acompañan a jóvenes y adultos con discapacidad intelectual. Obtenidas las autorizaciones institucionales pertinentes, se hizo extensiva la invitación a participar a la totalidad de trabajadores de ambas instituciones. Participaron un total de 35 trabajadores: 17 empleados del Centro de día MA y 18 del Centro de día JP. Se desarrollaron dos programas: uno para los trabajadores del Centros de Día MA (desde el mes de agosto a octubre de 2020) y otro para los de JP (desde el mes de octubre a diciembre del año 2020). La participación fue voluntaria y todos firmaron un consentimiento informado.

Para la evaluación pre y pos test se construyó un Formulario de Google creado a partir de la cuenta oficial de la Universidad del Aconcagua, en el cual se incluyeron tanto el consentimiento informado como los instrumentos seleccionados. Se respetaron los acuerdos de la Declaración de Helsinki.

El estudio constó de dos fases: 1. ${ }^{a}$ Evaluación (pre y pos test) y 2. ${ }^{a}$ Intervención. Los instrumentos utilizados en la evaluación pre y pos test fueron: el Cuestionario de Salud General (GHQ 12), el Índice de Felicidad de Pemberton, el Inventario de ansiedad de Beck (BAI), el Maslach Burnout Inventory (MBI) y una ficha sociodemográfica ad hoc.

\section{Análisis de resultados}

Para el cálculo de los resultados, se utilizó el paquete estadístico SPSS-21 (IBM Coorp). Obtenidos los datos descriptivos de las pruebas psicométricas empleadas, se exploró la normalidad de la distribución y se consideró un nivel de significancia estadística $\alpha \leq .05$. Para los datos que revelaron una distribución normal, se utilizó la prueba paramétrica $t$ de Student y para los que indicaron una distribución no normal se utilizó la prueba no paramétrica test de Wilcoxon. 


\section{Resultados}

Los datos descriptivos obtenidos por los participantes en los diferentes instrumentos utilizados en el estudio pueden verse en la tabla 2.

Tabla 2

Datos estadísticos descriptivos de los resultados pre y pos test obtenidos

\begin{tabular}{|c|c|c|c|c|c|c|}
\hline \multicolumn{2}{|l|}{ Instrumento } & \multirow{2}{*}{$\begin{array}{c}\text { Mínimo } \\
.00\end{array}$} & \multirow{2}{*}{$\begin{array}{c}\text { Máximo } \\
8.00\end{array}$} & \multirow{2}{*}{$\begin{array}{c}\text { Media } \\
5.25\end{array}$} & \multirow{2}{*}{$\begin{array}{c}\text { Desviación estándar } \\
1.83\end{array}$} & \multirow[t]{2}{*}{ Incremento } \\
\hline $\begin{array}{l}\text { Pretest Puntuación } \\
\text { Malestar-GHQ }\end{array}$ & Total & & & & & \\
\hline $\begin{array}{l}\text { Postest Puntuación } \\
\text { Malestar-GHQ }\end{array}$ & Total & .00 & 9.00 & 4.57 & 1.95 & -0.68 \\
\hline $\begin{array}{l}\text { Pretest Puntuación } \\
\text { Bienestar-PHI }\end{array}$ & Total & 52.00 & 97.00 & 83.65 & 9.39 & \\
\hline $\begin{array}{l}\text { Postest Puntuación } \\
\text { Bienestar-PHI }\end{array}$ & Total & 70.00 & 96.00 & 84.85 & 6.08 & +1.2 \\
\hline $\begin{array}{l}\text { Pretest Puntuación } \\
\text { Ansiedad-BAI }\end{array}$ & Total & 3.00 & 47.00 & 13.77 & 10.32 & \\
\hline $\begin{array}{l}\text { Postest Puntuación } \\
\text { Ansiedad-BAI }\end{array}$ & Total & .00 & 34.00 & 11.37 & 8.92 & -2.4 \\
\hline $\begin{array}{l}\text { Pretest Puntuación } \\
\text { AE-MBI }\end{array}$ & Total & 5.00 & 42.00 & 16.17 & 8.75 & \\
\hline $\begin{array}{l}\text { Postest Puntuación } \\
\text { AE-MBI }\end{array}$ & Total & 3.00 & 34.00 & 13.02 & 7.30 & -3.14 \\
\hline $\begin{array}{l}\text { Pretest Puntuación T } \\
\text { MBI }\end{array}$ & tal D- & .00 & 14.00 & 2.42 & 3.68 & \\
\hline $\begin{array}{ll}\text { Postest Puntuación } \\
\text { D-MBI }\end{array}$ & Total & .00 & 12.00 & 1.74 & 3.04 & -0.68 \\
\hline $\begin{array}{l}\text { Pretest Puntuación } \\
\text { RP-MBI }\end{array}$ & Total & 17.00 & 46.00 & 36.74 & 6.84 & \\
\hline $\begin{array}{l}\text { Postest Puntuación } \\
\text { RP-MBI }\end{array}$ & Total & 24.00 & 48.00 & 38.54 & 6.04 & +1.8 \\
\hline
\end{tabular}

A partir de los datos resultantes del cuestionario GHQ-12, tras la participación en el programa, se observó una disminución del malestar. Se hallaron diferencias estadísticamente significativas entre las mediciones pre y pos test $(Z=-2.86 ; p<.004)$. La reducción cuantitativa media observada en el pos test fue de 0.68 puntos; por lo tanto, es posible afirmar que la participación en el programa generó una disminución del malestar de los participantes (ver tabla 3). 


\section{Tabla 3}

Valores medios, desviación estándar (DE) y comparaciones entre los resultados pre y pos test en las diferentes pruebas

\begin{tabular}{|c|c|c|c|c|c|}
\hline Pruebas administradas & Media & $\mathrm{DE}$ & Media & $\mathrm{DE}$ & Z \\
\hline General Health Questionarire GHQ 12 & 5.25 & 1.83 & 4.57 & 1.95 & $-2.866^{*}$ \\
\hline Índice de Felicidad de Pemberton PHI & 83.65 & 9.39 & 84.85 & 6.08 & 0.89 \\
\hline Inventario de Ansiedad de Beck BAI & 13.77 & 10.32 & 11.37 & 8.92 & -1.872 \\
\hline $\begin{array}{l}\text { Maslach Burnout Inventory- MBI Agotamiento } \\
\text { emocional }\end{array}$ & 16.17 & 8.75 & 13.02 & 7.30 & $-2.660 *$ \\
\hline Maslach Burnout Inventory- MBI & 2.42 & 3.68 & 1.74 & 3.04 & -1.614 \\
\hline Despersonalización & & & & & \\
\hline $\begin{array}{l}\text { Maslach Burnout Inventory- MBI Realización } \\
\text { personal }\end{array}$ & 36.74 & 6.84 & 38.54 & 6.04 & 1.166 \\
\hline
\end{tabular}

${ }^{*} p \leq .05$

Del análisis de los resultados del Índice de Felicidad de Pemberton-PHI, tras su participación en el programa, se observó un incremento en el bienestar recordado sobre diversas áreas de su vida. El incremento cuantitativo medio observado en el pos test fue de 1.2 puntos. No obstante, los resultados obtenidos de la prueba no paramétrica de Wilcoxon no reflejaron diferencias estadísticamente significativas entre las mediciones pre y postest $(\mathrm{Z}=0.89 ; p>.373)$.

En cuanto al tercer instrumento utilizado, el BAI, se observó una disminución de la ansiedad tras la participación en el Programa. La reducción cuantitativa media observada en el pos test fue de 2.4 puntos. Sin embargo, los resultados obtenidos de la prueba no paramétrica de Wilcoxon no arrojaron diferencias estadísticamente significativas entre las mediciones pre y $\operatorname{pos}(\mathrm{Z}=-1.872 ; p>.061)$.

A partir de los datos resultantes del MBI, se observó que, si bien en la medición pre test solo un participante (3\%), cumplía los tres requisitos del síndrome de burnout (alto agotamiento emocional y despersonalización y baja realización personal en el trabajo) en la evaluación pos test. Ningún participante cumplía tales requisitos.

Se consideró oportuno analizar cada una de las subescalas del MBI y los resultados obtenidos se detallan a continuación. En relación con el AE del MBI, tras la participación en el programa, se observó una disminución del cansancio emocional de los participantes. La disminución cuantitativa media observada en el pos test fue de 3.14 puntos. Los resultados obtenidos de la prueba no paramétrica Wilcoxon test ofrecieron diferencias estadísticamente significativas entre las mediciones pre y pos test $(Z=-2.660$; $p<.008)$.

Tras la participación en el programa, se observó una disminución en la subescala D del MBI. En todo caso, la reducción cuantitativa media en el pos test fue de 0.68 puntos. No obstante, los resultados obtenidos de la prueba no paramétrica de Wilcoxon no indicaron diferencias estadísticamente significativas entre las mediciones pre y pos $(\mathrm{Z}=-1.614 ; p>.106)$. 
Por último, se observó un incremento en la realización personal en el trabajo (subescala RP del MBI) tras la participación en el programa. El incremento cuantitativo medio observado en el pos test fue de 1.8 puntos. Sin embargo, los resultados obtenidos de la prueba no paramétrica de Wilcoxon no arrojaron diferencias estadísticamente significativas entre las mediciones pre y pos $(Z=1.166 ; p>.244)$.

\section{Discusión}

Jané-Llopis (2004) asevera que "los programas que promocionan la salud mental no sólo funcionan, sino que además contribuyen a un mayor bienestar mental y aumentan la calidad de vida a nivel individual y comunitario" (p. 74). El presente estudio se propuso como objetivo valorar el impacto de un programa de promoción del bienestar psicológico adecuado a las condiciones de confinamiento debido a la pandemia del COVID-19 en Argentina. Las sesiones diseñadas y desarrolladas durante el aislamiento promovieron cambios estadísticamente significativos en dos de las variables dependientes estudiadas: malestar psicológico (GHQ 12) y agotamiento emocional (subescala del MBI). Esto permite inferir que, participar en este programa, resultó beneficioso para disminuir el malestar psicológico e incrementar la estabilidad emocional de los trabajadores.

Por un lado, las mediciones pos test reportaron cambios cuantitativos en las otras variables en estudio, de manera tal que se observó un incremento del bienestar y de realización personal en el trabajo. Por otro lado, hubo una disminución en la severidad y gravedad de la ansiedad y despersonalización, aunque tales cambios no alcanzaron una significancia estadística.

En tiempos de alerta sanitaria por la pandemia por COVID-19, el encuentro semanal regular durante los dos meses de duración de la intervención, con la terapeuta y los compañeros de trabajo, a través de una videoconferencia, parece haber resultado eficaz en la promoción del bienestar de los participantes.

Si bien los datos cuantitativos como los estadísticos permiten acreditar ciertos beneficios de la aplicación grupal, online sincrónica del Programa en cuestión, el tamaño y tipo muestral no permite generalizar los resultados. No obstante, cabe mencionar que, en contextos terapéuticos, este tipo de estudios con control parcial (Van Dalen \& Meyer, 1971) o no aleatorizado (Anderson et al., 1980) permiten una suficiente verificación de la variable independiente.

La situación sanitaria vivida en el año 2020 urgió implementar estrategias de intervención online, que fomentaron la interacción social y, al mismo tiempo, el cuidado de la propia salud de los trabajadores. En el siglo XXI, y especialmente debido a la pandemia que ha azotado a toda la humanidad, resulta prioritario promover la salud y reducir el padecimiento mental.

$\mathrm{Al}$ atender que la propuesta fue completamente online, desarrollada enteramente durante el tiempo de confinamiento y, por ende, de teletrabajo, sus resultados son sugerentes en lo que respecta a la aplicación de programas de promoción de la salud grupal, online sincrónico. El programa propuesto ha sido diseñado desde un encuadre cognitivo conductual, por lo que puede ser un aporte al estudio de la eficacia de tales abordajes: "Los tratamientos psicológicos dispensados a través de internet representan un modelo emergente para mejorar el acceso a las Terapias Cognitivo Conductuales basada en la evidencia" (Sandín et al., 2020, p. 197). 
Los hallazgos del presente estudio son alentadores: participar en un programa de promoción de la salud grupal, online, sincrónico, de 24 horas de duración ( 8 sesiones semanales), con énfasis en un estilo de vida saludable y en el entrenamiento en técnicas de regulación emocional, protegió a los trabajadores del impacto de los eventos estresantes asociados al confinamiento durante la pandemia.

\section{Referencias}

American Psychological Association (2013). Guidelines for the practice of telepsychology. https://www.apa.org/ pubs/journals/features/amp-a0035001.pdf

Anderson, S., Auquier, A., Hauchk, W.W., Oakes, D., Vandeale, W., \& Weisberg, H. (1980). Statistical methods for comparative studies. John Wiley.

Arnau, J., \& Gómez, J. (1995). Diseños longitudinales en panel. En J. Arnau (Ed.), Diseños longitudinales aplicados a las ciencias sociales y del comportamiento (pp. 54-72). Limusa-Noriega.

Arón, A. (2016, 8-11 de noviembre). Cuidar a los que trabajan: climas laborales tóxicos y nutritivos. [Ponencia]. XXI Congreso Internacional del CLAD sobre la Reforma del Estado y de la Administración Pública, Santiago, Chile. https://www2.congreso.gob.pe/sicr/cendocbib/con4 uibd.nsf/ FE7787B8E9EEBF22052580B200821538/\$FILE/aron.pdf

Arrigoni, F. (2021). Programa grupal, en línea, sincrónico para promover la salud y el bienestar psicológico durante la pandemia de COVID-19. En Enciclopedia Argentina de Salud Mental (6a ed.). http://www. enciclopediasaludmental.org. ar/trabajo.php?idt=130\&idtt $=224$

Arrigoni, F., \& Fadin, H. (2019). Cuidando a los que educan. Revista Academia, 18(42), 7-21. http://www.saber. ula.ve/handle/123456789/46331

Arrigoni, F., Morán, C., González De-Cicco, J., \& Polo, I. (2021). Eficacia de un programa de promoción de la salud para voluntarios. Revista Electrónica de Psicología Política, 19(46). http://www.psicopol.unsl.edu.ar/ pdf/REPP-A19-N46-Art01.pdf

Arrigoni, F., Navarro-Guzmán, J., \& Marchena-Consejero, E. (2020). Well-being and Health during COVID-19 Pandemic. En O. Titrek \& G. Sezen-Gultekin (Eds.), 6th International conference on lifelong education and leadership for all. Conference proceeding book (pp. 55-60). Sakarya University.

Barlow, D., Farchione, T., Sauer-Zavala, S., Murray Latin, H., Ellard, K., Bullis, J., Bentley, K., Boettcher, H., \& Cassiello-Robbins, C. (2019). Protocolo unificado para el tratamiento transdiagnóstico de los trastornos emocionales. Manual del paciente (2 $2^{\mathrm{a}}$ ed.). Alianza.

Beck, A., \& Steer, R. (2011). Manual for the Beck Anxiety Inventory. The Psychological Corporation.

Beecham, J., Bonin, E., Görlich, D., Baños, R., Beintner, I., Buntrock, C., Bolinski, F., Botella, C., Ebert, D., Herrero, R., Potterton, R., Riper, H. Schmidt, U., Waldherr, K., Weisel, K., Zarski, A., Zeiler, M., \& Jacobi, C. (2019). Assessing the cost and cost-effectiveness of ICare internet-based interventions (Protocol). Internet Interventions, 16, 12-19. https://doi.org/10.1016/j.invent.2018.02.009

Blanch, J. M. (2011). La psicología del trabajo ante la crisis del empleo. http://www.cop.es/infocop/pdf/2012.pdf 
EFICACIA DE UN PROGRAMA GRUPAL, ONLINE SINCRÓNICO DE SALUD Y BIENESTAR

Burrone, M., Abeldaño, A., Susser, L., Lucchese, M., Enders, J., Alvarado, R., Eliecer, V., \& Fernández, R. (2015). Evaluación psicométrica y estudio de fiabilidad del cuestionario general de salud (GHQ-12) en consultantes adultos del primer nivel de atención en Córdoba, Argentina. Revista de la Facultad de Ciencias Médicas, 73(3), 236-242.

Cabas-Hoyos, C. (2020). Intervenciones en salud mental apoyadas en tecnologías: la telepsicología. En P. Acero, K. Cabas, C. Caycedo, P. Figueroa, G. Patrick, G. \& M. Martínez-Rudas, Telepsicología. Sugerencias para la formación y el desempeño profesional responsable (pp. 43-62). ASCOFAPSI. https://ascofapsi.org.co/pdf/ Libros/Telepsicologia_web.pdf

Casari, L., Llano López, L., Moreno, E., Nosal, P., Piera, L., Torres, N., \& Waldheim, J. (2017). Burn Out y calidad de vida percibida en profesionales residentes del área materno-infantil de la provincia de Mendoza. Investigaciones en Psicología, 22(2), 7-16.

Consejo General de la Psicología de España (2020, 19 de marzo). Recomendaciones dirigidas a la población para un afrontamiento eficaz ante el malestar psicológico generado por el brote de coronavirus-Covid-19. Infocoponline. http://www.infocop.es/view_article.asp?id=8627

Consejo General de Psicología de España. (2017). Guía para la práctica de la telepsicología [Guidelines for the practice of telepsychology]. http://www.cop.es/pdf/telepsicologia2017.pdf

De la Torre-Martí, M., \& Pardo-Cebrián, R. (2018). Guía para la intervención en telepsicología. Colegio Oficial de Psicólogos de Madrid. https://www.psichat.es/guia-para-la-intervencion-telepsicologica-2019.pdf

De Witte, H. (2016). On the Scarring Effects of Job Insecurity (and How They Can Be Explained). Scandinavian Journal of Work, Environment \& Health, 27(2), 146-153. https://doi. org/10.5271/sjweh.3545

Delfino, G., Botero, C., \& González-Insua, F. (2019). Una escala corta de bienestar: el Índice de felicidad de Pemberton aplicado a población adulta de Buenos Aires. Anuario de investigaciones, XXVI, 115-122. http:// www.psi.uba.ar/investigaciones/revistas/anuario/trabajos_completos/26/delfino.pdf

Federación de Psicólogos de la República Argentina. (2021). Recomendaciones para las buenas prácticas mediante la utilización de TICS (tecnologías de la información y la comunicación). http://www.fepra.org. $\underline{\operatorname{ar} / 2021 / 11 / 09 / \text { recomendaciones-para-las-buenas-practicas-mediante-la-utilizacion-de-tics-tecnologias-de- }}$ la-informacion-y-la-comunicacion/

Fullerton, C., Acuña, J., Florenzano, R, Cruz, C., \& Weil, K. (2003). Psicopatología en pacientes hospitalizados en un hospital general. Revista chilena de Neuropsiquiatría, 41(2), 103-109.

Goldberg, D.P. \& Williams, P. (1988). Users' Guide to the General Health Questionnaire. NFER-Nelson.

Hein, N. (2005). Estudio del síndrome de burnout en pastores adventista y su relación con la satisfacción laboral y las características de personalidad [Tesis de Licenciatura inédita]. Universidad Adventista del Plata.

Hernández-Sampieri, R., \& Mendoza, C.P. (2018). Metodología de la investigación. McGraw Hill.

Hervás, G., \& Vázquez, C. (2013). Construction and validation of a measure of integrative well-being in seven languages: The Pemberton Happiness Index. Health \& Quality of Life Outcomes, 11, 66. http://doi. org/10.1186/1477-7525-11-66 
Hosman, C., Jané-Llopis, E., \& Saxena, S. (2005). Prevención de trastornos mentales: intervenciones efectivas y opciones de políticas. Oxford University Press.

IBM Coorp. SPSS Statistics 21.

Jané-Llopis, E. (2004). La eficacia de la promoción de la salud mental y la prevención de los trastornos mentales. Revista de la Asociación Española de Neuropsiquiatría, 89, 67-77.

Maslach C., \& Jackson, S. (1981). Maslach Burnout Inventory. Consulting Psychologist Press.

Organización Mundial de la Salud. (2020, 1 de julio). ¿Qué es una pandemia? https://www.who.int/csr/disease/ swineflu/frequently asked questions/pandemic/es/

Pemberton Happiness Index. Recuperado a partir de: http://www.pembertonindex.com

Pineda-Sánchez, D. (2018). Procesos transdiagnósticos asociados a los sintomas de ansiedad y depresión [Tesis doctoral, Universidad Nacional de Educación a distancia]. http://e-spacio.uned.es/fez/eserv/tesisuned:EDPg-PsiSal-Dpineda/PINEDA_SANCHEZ_DAVID_Tesis.pdf

Rosen,A., Gilld, N., \& Salvador-Carulla, L. (2020). The future of community psychiatry and community mental health services. Current Opinion in Psychiatry, 33, 375-390. https://doi.org/10.1097/YCO.0000000000000620

Sanclemente, F., Gamero, N., Arenas, A., \& Medina, M. (2020). Análisis de la satisfacción, la inseguridad y la salud laboral en población empleada durante la reciente crisis económica global. Anuario de Psicología, 50(3), 149-159. https://doi. org/10.1344/anpsic2020.50.14

Sandín, B., Valiente, R.M., García-Escalera, J., Pineda, D., Espinosa, V., Magaz, M., \& Chorot, P. (2020). Protocolo unificado para el tratamiento transdiagnóstico de los trastornos emocionales en adolescentes a través de internet (iUP-A): Aplicación web y protocolo de un ensayo controlado aleatorizado. Revista de Psicopatología y Psicología Clínica, 24,197-215. https://doi.org/10.5944/rppc.26460

Van Dalen, D.B., \& Meyer, W.J. (1971). Manual de técnica de la investigación educacional. Paidós.

Vera-Villarroel, P., Pavéz, P., \& Silva, J. (2012). El rol predisponente del optimismo: hacia un modelo etiológico del bienestar. Terapia Psicológica, 30(2), 77-84. https://www.scielo.cl/pdf/terpsicol/v30n2/art08.pdf

Vizioli, N., \& Pagano, A. (2020). Adaptación del Inventario de Ansiedad de Beck en población de Buenos Aires. Interacciones, 6(3), e171. https://doi.org/10.24016/2020.v6n3.171

Wood, A., \& Joseph, S. (2010). The absence of positive psychological (eudemonic) well-being as risk factor for depression: A ten years cohorte study. Journal of Affective Disorders, 122(3), 213-217. https://doi. org/10.1016/j.jad.2009.06.032 
Recibido: 09 de Marzo de 2021 Revisión recibida: 15 de Octubre de 2021

Aceptado: 28 de Octubre de 2021

\section{Sobre las autoras:}

Flavia Arrigoni es magíster en Intervención Psicológica en Contextos de Riesgo, profesora de la Universidad del Aconcagua (Argentina) y doctoranda de la Universidad de Cádiz (España). Trabaja como docente e investigadora en Argentina.

Mara Agustina Abraham es Licenciada en Psicología.

Ivana Polo es magíster en Criminología, Profesora de la Universidad del Aconcagua (Argentina). Labora como docente e investigadora en Argentina. 